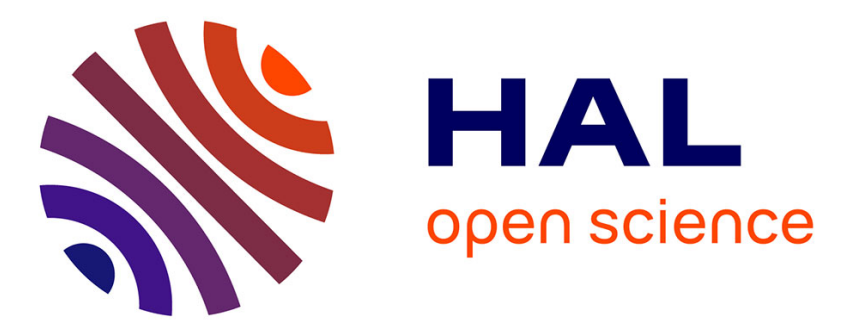

\title{
Gibbs free energy of liquid water derived from infrared measurements
}

\author{
Isabelle Bergonzi, Lionel Mercury, Jean-Blaise Brubach, Pascale Roy
}

\section{To cite this version:}

Isabelle Bergonzi, Lionel Mercury, Jean-Blaise Brubach, Pascale Roy. Gibbs free energy of liquid water derived from infrared measurements. Physical Chemistry Chemical Physics, 2014, 16, pp.24830 - 24830. 10.1039/c4cp03466j . insu-01090133

\section{HAL Id: insu-01090133 \\ https://hal-insu.archives-ouvertes.fr/insu-01090133}

Submitted on 8 Dec 2014

HAL is a multi-disciplinary open access archive for the deposit and dissemination of scientific research documents, whether they are published or not. The documents may come from teaching and research institutions in France or abroad, or from public or private research centers.
L'archive ouverte pluridisciplinaire HAL, est destinée au dépôt et à la diffusion de documents scientifiques de niveau recherche, publiés ou non, émanant des établissements d'enseignement et de recherche français ou étrangers, des laboratoires publics ou privés.

\section{(ㅇ)(1) $\$$}

Distributed under a Creative Commons Attribution - NonCommercial - NoDerivatives| 4.0 


\title{
Gibbs free energy of liquid water derived from infrared measurements
}

\author{
Isabelle Bergonzi ${ }^{1}$, Lionel Mercury ${ }^{1}$, Jean-Blaise Brubach $^{2}$, Pascale Roy $^{2}$
}

1. Institut des Sciences de la Terre d'Orléans, UMR 7327 Université d'Orléans/ CNRS/ BRGM, 45071 Orléans cedex, France

2. Synchrotron SOLEIL, L’Orme des Merisiers, Saint Aubin, BP 489, 91192 Gif-sur-Yvette, France.

Keywords: Liquid water; Vibrational energy; partition function; Gibbs free energy.

\begin{abstract}
Infrared spectra of pure liquid water were recorded from $20 \mathrm{~cm}^{-1}$ to $4000 \mathrm{~cm}^{-1}$ at temperatures ranging from $263 \mathrm{~K}$ to $363 \mathrm{~K}$. The evolution of connectivity, libration, bending and $\mathrm{OH}$ stretching bands as a function of temperature follows the evolution of the inter-molecular dynamics, and so gives insight into the internal energy averaged over the measurement time and space. A partition function, which takes into account the inter-molecular and intra-molecular modes of vibration of water, all variable with the molecular networking, was developed to convert this vibrational absorption behavior of water into its macroscopic Gibbs free energy, assuming the vibrational energy to feature most of the water energy. Calculated Gibbs free energies along the thermal range are in close agreement with the literature's values up to $318 \mathrm{~K}$. Above this temperature, contributions specific to the non H-bonded molecules must be involved to closely fit the thermodynamics of water. We discussed this temperature threshold in relation to the wellknown isobestic point. Generally speaking, our approach is valuable to convert the IR molecular data into mean field properties, quantitative basis to predict how water behaves in natural or industrial settings.
\end{abstract}




\section{INTRODUCTION}

Liquid water is present in abundance on and inside Earth. It is found, most of time, under its more common form, usually called the "bulk form". Its physico-chemical properties ${ }^{1,2}$ are governed by the dense network of hydrogen bonds ${ }^{3}$ (H-bond) that one water molecule establishes with its close neighbours. Nevertheless, in the natural systems, liquid water can be found in other exotic forms, for instance solvation water, interfacial water layers located at the direct contact to minerals surface or water confined in restricted spaces (various types of pores). These varying forms of liquid water exhibit intrinsic properties that deviate from those of bulk water ${ }^{4-11}$. Consequently, their reactive properties with the surrounding matter (minerals, organics, gases, proteins) are also different to those of bulk water, making the by-products of this reactivity very different (pollutants, for instance, mobility/immobility; water sustainability, etc), especially on the long-term. The geochemical cycles in nature could be seriously reconsidered if a thermodynamic typology of liquid could be established with respect to a typology of the containing reservoir (atmosphere, rivers, soils, surfacial to deep aquifers, deep crust).

To reach this goal, it is necessary to have a method for measuring these reactive properties while preserving the characteristics of the wide variety of possible containers. Infrared (IR) spectroscopy is a technique of choice to probe the strength of the H-bonds between water molecules, even trapped inside different types of materials, averaging the signal on micrometric distances and "long" time. It has been widely used to probe the vibrational properties of water ${ }^{13-15}$ even in its exotic forms ${ }^{16-23}$ or for reduced amount of matter, demonstrating its ability to record small energetic changes of water.

Nevertheless, the widely known relationship between vibrational properties and energetics of water is not quantified in terms of macroscopic thermodynamic properties. Some studies ${ }^{24-26}$ proposed a statistical deconvolution of the IR band to thermodynamic values but they are only available for diluted solutions of HOD molecules in $\mathrm{D}_{2} \mathrm{O}\left(\right.$ or $\left.\mathrm{H}_{2} \mathrm{O}\right)$ and therefore cannot describe the IR spectrum 
of bulk (normal) water. Moreover, this type of approach only takes into account the $\mathrm{OH}$ stretching band of water, while the infrared spectrum of liquid water is composed of four characteristic bands ranging from approximately $100 \mathrm{~cm}^{-1}$ to $4000 \mathrm{~cm}^{-1}$. The stretching band centered at $3400 \mathrm{~cm}^{-1}$ is the most intense and is very sensitive to the inter-molecular environment of water molecule, and is commonly used as a probe of the water networking. Alternative approach used a wider energy range, for interpreting spectra measured in the Attenuated Total Reflection optical configuration ${ }^{15}$. We choose here to only exploit transmission spectra, as it allows extracting reliable values for absorbance without assumptions on incidence angles, refractive index and geometric limitations.

In this paper, the existing partition function from Giffiths and Scheraga ${ }^{27}$ is modified to convert the IR-absorption properties of bulk water into Gibbs free energy values, and this conversion is tested against the well-known thermodynamic values as a function of temperature. For this purpose, a new set of IR bands of water, from the connectivity band to the $\mathrm{OH}$ stretching bands reported from $263 \mathrm{~K}$ to $363 \mathrm{~K}$, is used to retrieve the Gibbs free energies. The IR-thermodynamic conversion shed light on some specific properties (e.g. isobestic point) related to the evolution of bulk liquid water with temperature.

\section{THE IR-to-THERMODYNAMICS CONVERSION}

Griffith and Sheraga ${ }^{27}$ devised a partition function from an original version designed by Nemethy and Sheraga ${ }^{28}$, able to transform vibrational data into thermodynamic properties. It is based on the "Flickering clusters" concept ${ }^{29}$ which assumes the existence of clusters of bonded water molecules, with different sizes, the H-bonds of which break and re-form continually. The model also implies that the clusters are surrounded by non H-bonded molecules and so the partition function is expressed as the addition of two main structures: the non H-bonded molecules and the clusters, this latter being distinguished into 1-, 2-, 3-, 4-bonded water species. The partition function is therefore focusing on the coordination number of water species (four water clusters and the non H-bonded molecules) in an analytical approach completely compatible with the percolation model ${ }^{30}$, a 
Revised version of the ID CP-ART-08-2014-003466. Submitted to Physical Chemistry Chemical Physics. September $22^{\text {th }}, 2014$.

statistical viewpoint of the water energetics. Hence, this function is well adapted to the objective of turning the IR information into mean field thermodynamic properties, assuming they are strictly determined by the mean connectivity of water molecules.

An energy level is ascribed to each species, and the molecules distribution between the energy levels is determined by the corresponding Boltzmann factors, $\exp \left(E_{i} / k T\right)$ where $E_{i}$ is the energy of a given energy level and $k$ is the Boltzmann constant, and by the degrees of freedom allowed for the motion of each species. The partition function can be written:

$Z=\sum_{N_{i}} g \prod_{i=0}^{4}\left[\zeta_{i} f_{i} \exp \left(-E_{i} / R T\right)\right]^{x_{i} N_{A}}$

Where $g$ is a combinatorial factor given by $=\frac{N_{A} !}{N_{0} ! N_{1} ! N_{2} ! N_{3} ! N_{4} !}$, with $N_{A}$ the Avogadro number and $N_{i}=x_{i} N_{A}$ with $\sum N_{i}=N_{A}$ are the number of each species per mole of water; $x_{i}$ are the mole fraction of each species; $E_{i}$ are the energy level of each species; $\zeta_{i}$ are a multiplicity factor equal to $1,4,6,4,1$ successively for $\mathrm{i}=0,1,2,3,4 ; f_{i}$ are the weighting factors describing the vibrational, rotational and translational freedom allowed to each species, ascribed from IR and Raman experimental data. The equation to calculate these $f_{i}$ factors writes:

$f_{i}=\left[1-\exp \left(-h v_{i}^{(T)} / k T\right)\right]^{-3}\left[1-\exp \left(-h v_{i}^{L} / k T\right)\right]^{-3}$

Where $v_{i}^{(T)}$ is the frequency describing the three degenerated translational degrees of freedom and $v_{i}^{(L)}$ the three degenerated restricted rotations, both pertaining to the $i^{\text {th }}$ energy level. The authors assumed that for each bonded state, all three translational modes are equal and all three restricted rotational modes are equal. The corresponding frequencies can be extracted from spectroscopic data, directly or indirectly. The authors assign a priori only the translational $\left(210 \mathrm{~cm}^{-1}\right)$ and the librational $\left(750 \mathrm{~cm}^{-1}\right)$ frequencies to the four-bonded molecules, based on the analogy with ice. The other values are chosen by a trial-and-error method to obtain the best fit of the theoretical thermodynamic quantities to the experimental ones. The values of $E_{i}(i=0,1,2,3)$, with $E_{4}=0$ being the ground state, are determined through the same procedure. 
Revised version of the ID CP-ART-08-2014-003466. Submitted to Physical Chemistry Chemical Physics. September $22^{\text {th }}, 2014$.

This model neglects the intra-molecular modes arguing that they are also present in the vapor phase, and so are not characteristic of the liquid phase. However, the shape of the intra-molecular bands, notably the $\mathrm{OH}$-stretching one, are very different between the liquid (continuous wide band) and the vapor (discontinuous peaks over a large frequency range) phases, expressing specificities in the energetics of these two phases. Consequently, we modified the partition function to include all the IR modes in the conversion calculations. The exact modifications introduced in the partition function will be detailed and justified further on. At this point, two facts should be emphasized: i) IR data can be converted into thermodynamic data using the intra- and inter-molecular measured frequencies; ii) the connectivity level of the molecules (from 0- to 4-bonded population) must be known as exactly as possible.

\section{MATERIALS AND METHODS}

We have recorded the IR spectra of liquid water at different temperatures. Three series of measurements were performed for different temperature ranges: the first report Mid-IR (MIR hereafter) spectra of water in an optimized environment under vacuum conditions; the second, also in MIR, uses a classic setup working at laboratory atmosphere; and the third extended the measurements to the low-frequency domain (Far-IR domain, noted FIR), using synchrotron radiation in a vacuum environment.

\subsection{MIR experiments under vacuum (SOLEIL)}

IR measurements, over the $400 \mathrm{~cm}^{-1}$ to $4000 \mathrm{~cm}^{-1}$ domain, were performed at SOLEIL Synchrotron at the AILES beamline using a Glowbar $\mathrm{SiC}$ source heated at $1200^{\circ} \mathrm{C}$. Spectroscopic investigations were performed using a Fourier Transform infrared spectrometer (IFS 125 HR, Bruker Instruments) combined with a $\mathrm{KBr}$ beamsplitter and a helium cooled bolometer detector. The spectra were recorded with a resolution of $4 \mathrm{~cm}^{-1}$ with 400 scans per spectrum. Transmission spectra were acquired using a copper fluid cell equipped with two diamond windows. The thickness of the liquid 
Revised version of the ID CP-ART-08-2014-003466. Submitted to Physical Chemistry Chemical Physics. September $22^{\text {th }}, 2014$.

film was fixed by the thickness of the Mylar spacer ring $(\approx 6 \mu \mathrm{m})$ and the sealing was ensured by two O-rings located respectively below and above the sandwich made up by the two windows and the liquid in between. The infrared coefficient absorption is defined by: $A=-\log \left(\frac{I}{I_{0}}\right)$, where $I_{0}$ is the transmitted intensity of the empty cell and $I$ the transmitted intensity of the cell filled with water. The fluid cell was fixed to a closed cycle helium cryostat allowing a temperature control down to $4.2 \mathrm{~K}$. The temperature ramp was controlled using the software SELIA in the $275 \mathrm{~K}$ to $350 \mathrm{~K}$ range.

\subsection{MIR experiments under atmospheric condition (ISTO)}

The measurements and the subsequent conversion were verified by measuring equivalent spectra using a Fourier Transform Infrared Micro-Spectrometer (Nicolet Continuum, Thermo Scientific). Notice that this laboratory setup allows a wider thermal range (263K - 363K) than the Soleil setup. In the following, we limit the discussion to the 275 - $350 \mathrm{~K}$ temperature range, and report the other data and the comparison between the two datasets in a supplementary material.

IR spectra were recorded, in the $850 \mathrm{~cm}^{-1}$ to $4000 \mathrm{~cm}^{-1}$ spectral range, using the laboratory spectrometer equipped with a XT-KBr beam splitter and a liquid nitrogen cooled Mercury Cadmium Telluride (MCT) wide range detector $(250 \mu \mathrm{m})$. The microscope runs in confocal mode, using a 15X infinity corrected Schwarzschild objective and a matching $15 \mathrm{X}$ condenser.

To prevent saturating the IR absorption, a fluid cell of varying thickness, down to the micrometer, was developed. This cell is made of two steel parts compressing two ZnSe windows (13 mm x 2 $\mathrm{mm}$ ), sealed with a fluorocarbon O-ring. A liquid drop was sandwiched and tightened between the two windows to form a bulk water film roughly $1 \mu \mathrm{m}$ thick. The cell was introduced in a heatingcooling stage (THMS-600, Linkam) compatible with IR light, controlling temperature at $\pm 0.1 \mathrm{~K}$.

Each spectrum was recorded with a resolution of $4 \mathrm{~cm}^{-1}$ with 200 scans per spectrum and no mathematical correction was used. The infrared coefficient absorption is defined as in the previous section. The spectra were recorded from $263 \mathrm{~K}$ (slightly supercooled state) to $363 \mathrm{~K}$. 


\subsection{FIR experiments with synchrotron source radiation}

Water in the FIR / THz regions $\left(600-50 \mathrm{~cm}^{-1}\right)$ was investigated using the SOLEIL Synchrotron radiation collected at the AILES beamline. The FTIR IFS 125HR spectrometer was combined with a $6 \mu \mathrm{m}$ Mylar beamsplitter and a helium cooled bolometer detector. For the THz $\left(100-10 \mathrm{~cm}^{-1}\right)$ measurements, the FTIR was combined with a $125 \mu \mathrm{m}$ Mylar beamsplitter and a $1.6 \mathrm{~K}$ helium pumped bolometer. All spectra were recorded with a resolution of $4 \mathrm{~cm}^{-1}$ with 400 scans per spectrum. Transmission spectra were acquired using a fluid cell made of copper and completed with two diamond windows. The thickness of the liquid film determined by the thickness of the Mylar spacer ring $(\approx 5 \mu \mathrm{m})$ and the sealing was insured by two O-rings located respectively below and above the stacking made up by the two windows and the liquid in the middle. The infrared coefficient absorption is defined as in the previous section. The fluid cell temperature was fixed to a helium closed cycle cryostat under vacuum (Model PT405 from CryoMech.) allowing a fine control of the temperature.

\section{RESULTS}

\subsection{Description of the bands}

The evolution of the IR bands in this spectral domain has been detailed by Brubach et $\mathrm{al}^{31}$ from $264 \mathrm{~K}$ to $320 \mathrm{~K}$. The present temperature range is wider, but the successive shape and absorption of the different bands meet their conclusions. Baseline correction is processed the same way for all spectra, using the Matlab function "backcor" developed by Mazet et al ${ }^{33}$, and the background is estimated by an asymmetric truncated quadratic cost function.

The bending mode mostly reflects water molecules that do not lie in a symmetric tetrahedral environment $^{31}$ as in ice. The band (Fig. 1A) exhibits an increasing absorbance with temperature, and the maximum slightly shifts toward lower wavenumbers from $1650.7 \mathrm{~cm}^{-1}$ at $263 \mathrm{~K}$ to 1648.3 $\mathrm{cm}^{-1}$ at $363 \mathrm{~K}$ (experiment under atmospheric condition) and from $1651.7 \mathrm{~cm}^{-1}$ at $275 \mathrm{~K}$ to $1646 \mathrm{~cm}^{-1}$ 
Revised version of the ID CP-ART-08-2014-003466. Submitted to Physical Chemistry Chemical Physics. September $22^{\text {th }}, 2014$.

at $350 \mathrm{~K}$ (experiment under vacuum). The shape of the band does not change much with temperature, except for a decreasing FWHM that agrees with the trends predicted by molecular modeling ${ }^{34}$. The increasing absorbance of the bending band with temperature is attributed to a decreasing number of water molecules in symmetric tetrahedral environment.

The stretching band is the most sensitive to any changes in the water network as its shape and frequency range is known to vary with the hydrogen bonds strength ${ }^{36}$. Also, the asymmetric shape of the band is usually interpreted as a convolution of several sub-bands, each pertaining to water molecules differently bonded to their close neighbors. At first sight, the maximum of the band (Fig. 1B) shifts toward higher wavenumbers as temperature increases (from $3380.6 \mathrm{~cm}^{-1}$ at $263 \mathrm{~K}$ up to $3436.5 \mathrm{~cm}^{-1}$ at $363 \mathrm{~K}$ for experiment under atmospheric condition and from $3390.3 \mathrm{~cm}^{-1}$ at $275 \mathrm{~K}$ to $3431.7 \mathrm{~cm}^{-1}$ at $350 \mathrm{~K}$ for experiment under vacuum). Simultaneously, the intensity of the band maximum decreases (from 0.82 a.u at $263 \mathrm{~K}$ to 0.61 a.u. at $363 \mathrm{~K}$ for experiment under atmospheric condition and from 0.85 a.u. at $275 \mathrm{~K}$ to 0.61 a.u. at $350 \mathrm{~K}$ for experiment under vacuum). Moreover its shape varies, losing the lower wavenumbers and enriching to higher ones with the FWHM increasing by about $6.5 \%$ with temperature. Finally, an isobestic-type crossing zone is displayed at high frequencies (see below, section 4.4). 

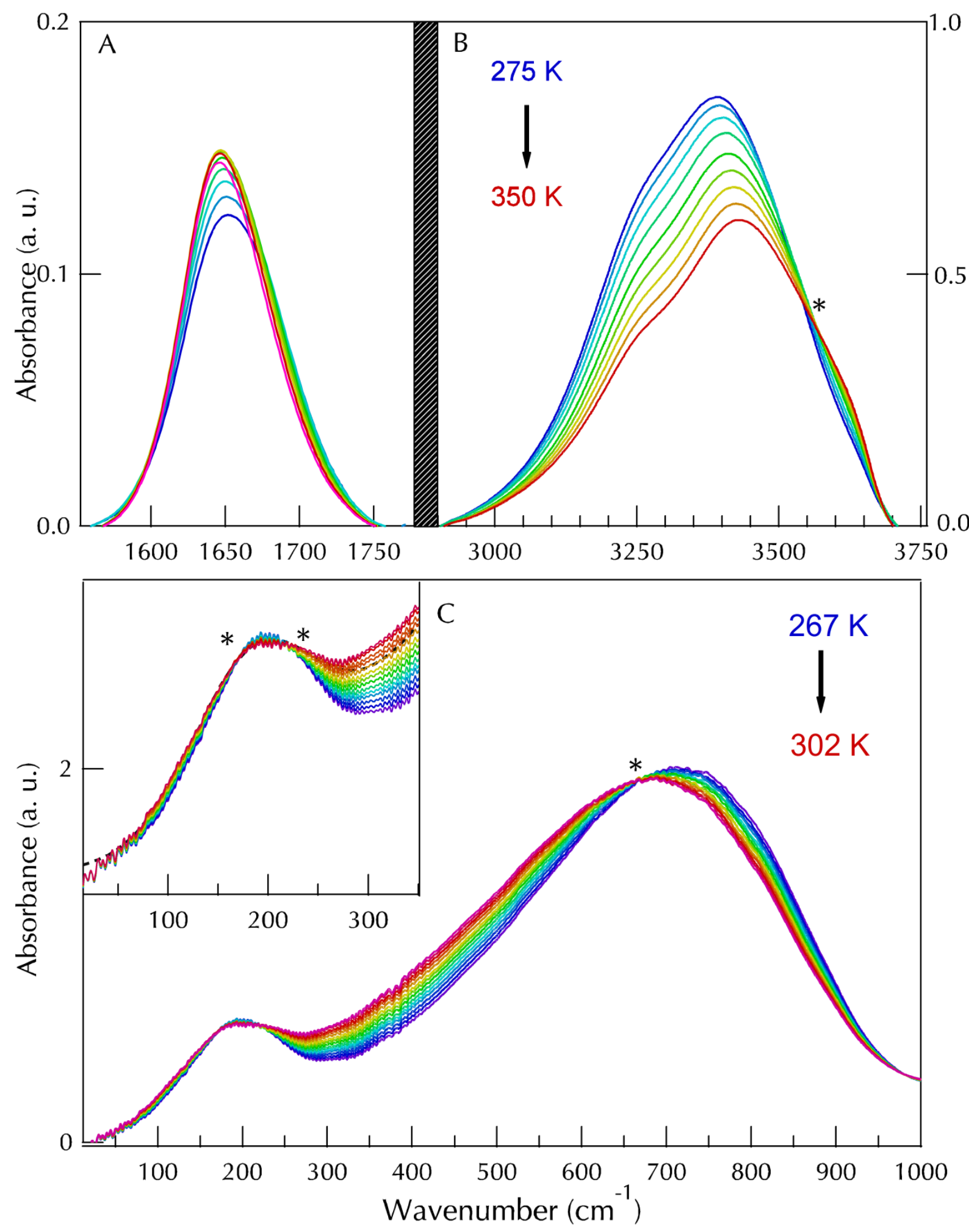

Figure 1. IR absorption bands of liquid water as a function of temperature (MIR experiment at Soleil): A. Bending, B. OH stretching, C. libration and connectivity bands. Stars outline the isobestic points (see text). 
Revised version of the ID CP-ART-08-2014-003466. Submitted to Physical Chemistry Chemical Physics. September $22^{\text {th }}, 2014$

Concerning the intermolecular bands, their intensity relates to the number of H-bonds rather than to the number of water molecules as for the intra-molecular modes. As a consequence, these intermolecular motions forcibly involve supramolecular assemblies: this system can be considered as a super molecule corresponding to H-bonded water molecules. Since the energies corresponding to these noncovalent bonds are one or two orders of magnitude lower than those of covalent bonds, their spectral signatures range at lower wavenumbers.

The libration band is ascribed to the frustrated rotations of rigid water molecules that are influenced by a force constant due to the H-bond between water molecules. These rotations have a vibrational character and are called librations. The corresponding band recorded using SR (Fig. 1C) and the one recorded from $275 \mathrm{~K}$ to $250 \mathrm{~K}$ with Glowbar source are in agreement with previous observations ${ }^{13,35}$ (although the latter was cutoff at $426 \mathrm{~cm}^{-1}$ ): it shows a decreasing intensity with temperature, and the main peak moves toward lower frequencies from $731.8 \mathrm{~cm}^{-1}$ at $275 \mathrm{~K}$ to $681 \mathrm{~cm}^{-1}$ at $350 \mathrm{~K}$. These changes demonstrate that the hindered rotations are temperature-sensitive, through the modifications of the molecular network of water. However, as already concluded ${ }^{31}$, the small shape changes with temperature suggest that the frustrated rotation is little or not affected by the number of $\mathrm{H}$ bonds established by the super molecule. From $267 \mathrm{~K}$ to $302 \mathrm{~K}$ (Fig. 1C), the libration band is complete and displays also a shift toward the lower frequencies: from $711.6 \mathrm{~cm}^{-1}$ at $267 \mathrm{~K}$ to 687.9 $\mathrm{cm}^{-1}$ at $302 \mathrm{~K}$, while the absorbance of the band decreases by $3 \%$ with temperature. An isobestic points is also shown in this temperature range (see below, section 4.4).

The connectivity band (Fig. 1C) is related to the intermolecular stretching vibration between two Hbonded water molecules. It is described as a translational stretching transition as opposed to the rotational like transition of the libration. From $267 \mathrm{~K}$ to $302 \mathrm{~K}$ the absorbance of the band decreases by 0.4 a.u. and the maximum is shifted toward the lower wavenumbers. However, owing to the high resolution of the band gained through the synchrotron source, the connectivity band appears less complex than usually proposed ${ }^{31,40}$. Its shape is quite regular, and can be fitted by a unique Gaussian sub-band. The observed variations (shape, frequency) with the temperature are very small, 
Revised version of the ID CP-ART-08-2014-003466. Submitted to Physical Chemistry Chemical Physics. September $22^{\text {th }}, 2014$.

especially compared to the other bands, and only the intensity of the connectivity is changing with $T$. Nonetheless, two isobestic points appear at the high- and low-frequencies sides of this band (see below, section 4.4).

\subsection{Decomposition of the stretching band}

Among all bands, the $\mathrm{OH}$ stretching band appears as the most sensitive probe of the $\mathrm{H}$-bonds network between water molecules. The oscillator strength of water molecules decreases when their coordination number increases and the corresponding $\mathrm{OH}$ frequency is downshifted ${ }^{36}$. In particular, the band frequency increases with the average distance between oxygen atoms of water molecules linked by $\mathrm{H}$-bond ${ }^{36}$, and the width of the band gives an averaged image of the H-bonds percolation throughout the molecular network.

To account for the various environments of a molecule, the $\mathrm{OH}$ stretching band is frequently decomposed into three Gaussian components ${ }^{16,18,20,31,37-40}$, each embodying a certain connectivity of the corresponding water molecules, the whole featuring the liquid in the experimental time and space. Hence, each Gaussian sub-band describes the quantity and the energy of the water molecules with a given mean coordination number (Fig. 2). The low frequency Gaussian is associated to the fully tetrahedrally coordinated water molecules, called network water (NW) also known as ice-like water since the Gaussian peak is close to its ice counterpart $\left(3250 \mathrm{~cm}^{-1}\right)$. The high-frequency Gaussian sub-band is ascribed to the molecules with a low coordination number (two, one or zero), called multimer water (MW) and close to the IR frequency of vapor molecules. The Gaussian subband lying in-between has a coordination number close to three, this population is called intermediate water (IW). 

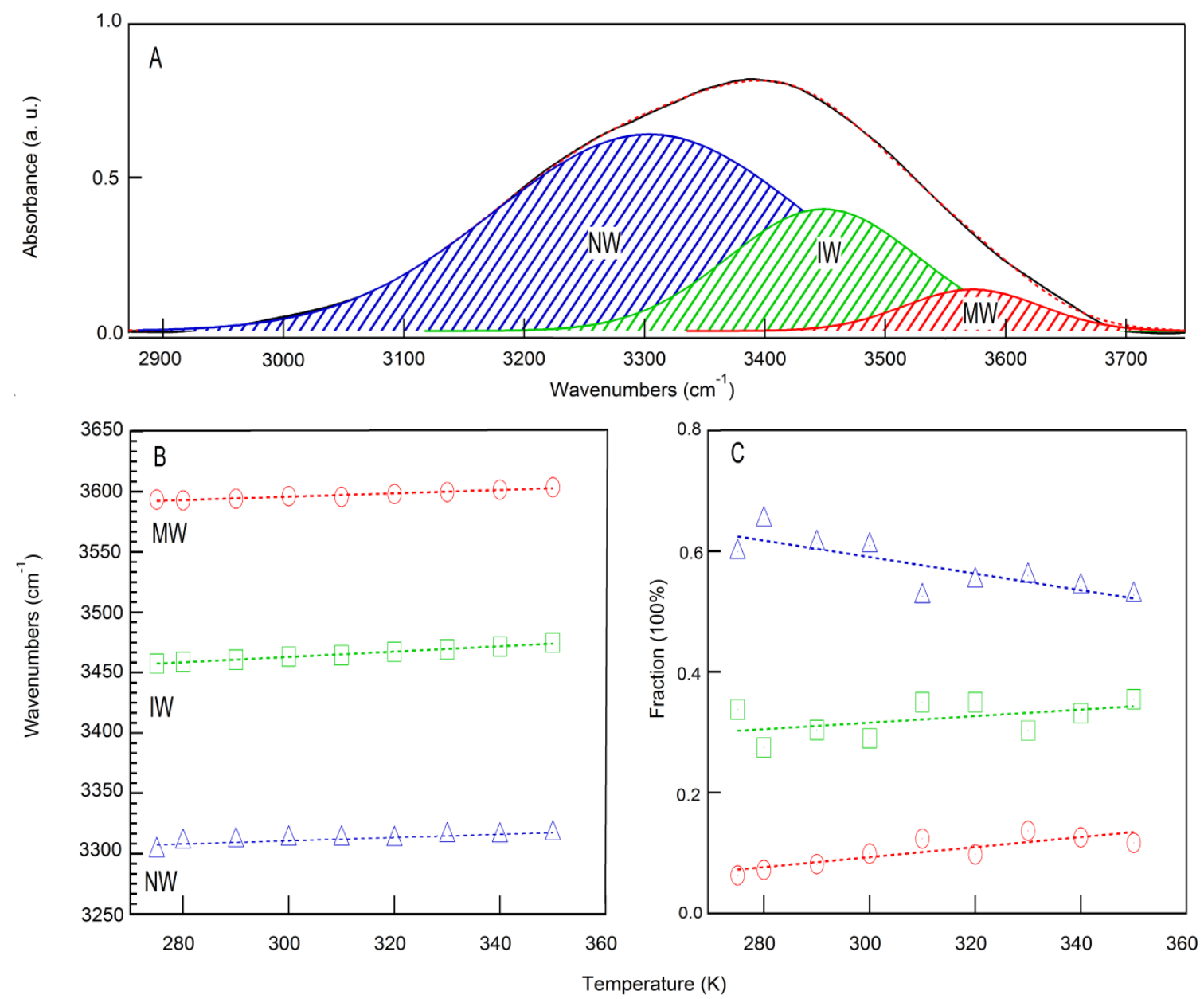

Figure 2. Upper, OH-stretching band at room temperature and its three Gaussian sub-bands, featuring the respective percentage of molecules with a given coordination number, averaged inside the probed volume and during the experimental time. Lower left, Frequency of each sub-bands as a function of temperature. Lower right, Molecular fractions of the three water populations (network water NW; intermediate water IW; multimer water MW; see text) as a function of temperature.

The experimental bands are free-fitted (Fig. 2) with the software Igor and its Multipeak fitting 2.0 package, to obtain the molecular fraction of each water population as a function of temperature. The molecular fraction of one population given by the ratio between the area of the Gaussian associated to the water population and the total area of the three Gaussians are reported in Table 1.

Along the temperature range, the NW fraction dominates the other two populations (Table 1, Fig. 2 lower right). The NW fraction however, decreases when temperature increases, while both the IW 
and MW fractions increase with temperature, as expected when liquid comes closer to the vapor phase transition. These changes clearly evidence a lowered connectivity of the H-bonded network as the boiling point is approached. These variations of the three populations are in agreement with previous studies ${ }^{31,37}$. Moreover, the mean coordination number is equal to $3.4 \pm 0.1$ at $298 \mathrm{~K}$, in agreement with numerical $^{41,42}$ and experimental ${ }^{37,43}$ studies.

The positions of the three Gaussians (Table 1, Fig. 2 lower left) are slightly shifted toward higher frequencies with the increasing temperature.

Table 1. Molecular fractions and main peak location of the sub-bands fitted on the $\mathrm{OH}$-stretching band recorded as a function of temperature (Soleil setup, AILES beamline).

\begin{tabular}{|c|c|c|c|}
\hline Water classes & Temperature & Molecular fractions & Main peak frequency $\left(\mathrm{cm}^{-1}\right)$ \\
\hline \multirow{3}{*}{ NW } & $275 \mathrm{~K}$ & 0.599 & 3303 \\
\hline & $350 \mathrm{~K}$ & 0.528 & 3317 \\
\hline & Variation & $-0.095 \% / K$ & $+0.19 \mathrm{~cm}^{-1} / \mathrm{K}$ \\
\hline \multirow{3}{*}{ IW } & $275 \mathrm{~K}$ & 0.338 & 3458 \\
\hline & $350 \mathrm{~K}$ & 0.355 & 3475 \\
\hline & Variation & $+\mathbf{0 . 0 2 2 7 \% / K}$ & $+0.23 \mathrm{~cm}^{-1} / \mathrm{K}$ \\
\hline \multirow{3}{*}{ MW } & $275 \mathrm{~K}$ & 0.063 & 3593 \\
\hline & $350 \mathrm{~K}$ & 0.117 & 3604 \\
\hline & Variation & $+0.072 \% / K$ & $+0.15 \mathrm{~cm}^{-1} / \mathrm{K}$ \\
\hline
\end{tabular}

The frequency shift of the maximum is clearly linked to a loss of order in the water molecular network $^{31,40}$ : an increasing temperature disrupts the connectivity and reduces percolation in liquid water. The associated variation of the band shape is consistent with this interpretation: the increasing intensity towards high wavenumbers and a loss towards low wavenumbers imply an increased fraction of the non H-bonded population and a decreased fraction of the connected molecules. 
Revised version of the ID CP-ART-08-2014-003466. Submitted to Physical Chemistry Chemical Physics. September $22^{\text {th }}, 2014$.

\subsection{Decomposition of the connectivity and libration bands}

From $267 \mathrm{~K}$ to $302 \mathrm{~K}$ the connectivity and libration bands are not fully resolved, and then require to be treated together: the whole ensemble was fitted by three Gaussian components, one for the connectivity band (Gauss 1) and two for the libration bands (Gauss 2 and Gauss 3, see Fig. 3).

The frequency of the connectivity band (Gauss 1) is almost constant with temperature (from 192.1 $\mathrm{cm}^{-1}$ at $267 \mathrm{~K}$ to $182.3 \mathrm{~cm}^{-1}$ at $301 \mathrm{~K}$, see Fig $3 \mathrm{~B}$ ), while its intensity decreases as expected from the loss of H-bonding with temperature. Concerning the libration band components (Gauss 2 and 3), their frequencies shift toward lower wavenumbers with temperature (Fig. 3B). In contrast, the intensity of Gauss 2 and 3 moves in an opposite way: Gauss 2's decreases with $T$, but Gauss 3's surprisingly increases with $T$. A close examination however shows that the total intensity of the libration (Gauss 2 and 3) decreases slightly (Fig. 1). Therefore, the increasing intensity of Gauss 3 is related to the fitting procedure encompassing a wider distribution of states when $T$ increases. 

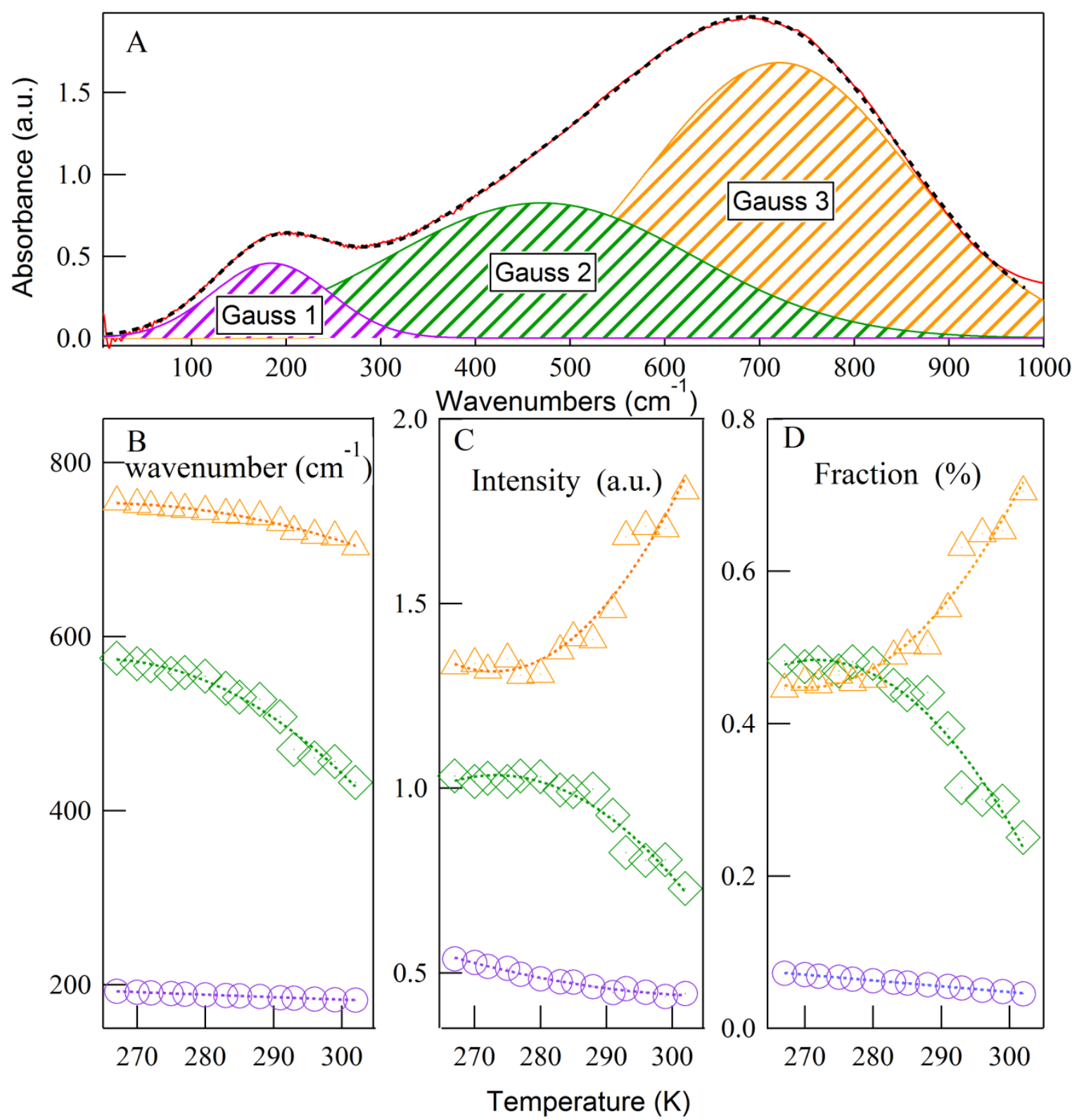

Figure 3. Upper, decomposition of the connectivity (one sub-band) and libration (two sub-bands) bands, here exemplified at $280 \mathrm{~K}$. Lower, evolution of the frequency, intensity and Gaussian subbands area as a function of temperature.

\subsection{Isobestic points}

As often observed in water vibrational spectroscopies (e.g. 31, 44), the $\mathrm{OH}$ stretching band spectra measured at various temperatures cross each other in an "isobestic" point at around $3500 \mathrm{~cm}^{-1}$. The term "isobestic" refers to the wavelength for which all absorption curves cross each other, i.e., 
Revised version of the ID CP-ART-08-2014-003466. Submitted to Physical Chemistry Chemical Physics. September $22^{\text {th }}, 2014$

where the absorbance is a constant. It implies some equilibrium between two absorbers. In previous studies (e.g., 37, 44, 46), this point was interpreted as the signature of two species of water molecules: H-bonded molecules and non-H-bonded molecules. Observing that the IW Gaussian sits very close to the isobestic point frequency, Brubach et al. ${ }^{31}$ reformulated this view in terms of balance between molecules that participate to the extended connective networks and those lying outside these networks.

This isobestic point as a function of temperature does not appear as a point but in a restricted area of frequency. Indeed in the temperature range of $263 \mathrm{~K}-313 \mathrm{~K}$, the spectra cross each other over frequencies spanning from 3520 to $3543 \mathrm{~cm}^{-1}$ (see supplementary material). Consequently, after other authors ${ }^{31}$, we designated it as a quasi-isobestic point. It is noteworthy here that this isobestic area is limited to a more restricted thermal range than previously observed ${ }^{44-47}$. The experiments carried out in atmosphere and under vacuum recorded such quasi-isobestic point $\left(\sim 3535 \mathrm{~cm}^{-1}\right)$ from $263 \mathrm{~K}$ to $313 \mathrm{~K}$. At higher temperatures $(310 \mathrm{~K})$, a vanishing of the quasi-isobestic crossing point with temperature ${ }^{44-47}$, is observed. Above this temperature, spectra intersections spread over $55 \mathrm{~cm}^{-1}$ for both series of measurements (see supplementary material), supporting a loss of the quasiisobestic point above $310 \mathrm{~K}$ in contrast with the previously-reported value of $353 \mathrm{~K}^{37}$.

In terms of raw data, the consistency of the present results measured using two different setups indicates that the observed disappearance does not depend on the experimental procedure. To go further in the interpretation, the Gaussian decomposition showed that the mean molecular network of water molecules is disturbed by the increasing temperature, despite that the NW remains the dominating population. This implies that water molecules maintain a strong connectivity even at high $T$, in agreement with previous studies ${ }^{3,48-50}$. Other effects may however come into play prompting us to re-interpret this vanishing point in the thermodynamic frame, involving the whole vibrational spectrum of liquid water, as developed below (see section 5.4).

A close examination of the low-frequency domain actually reveals three other isobestic points. The $669 \mathrm{~cm}^{-1}$ one, appearing within the libration band in agreement with previous measurements ${ }^{15}$, 
Revised version of the ID CP-ART-08-2014-003466. Submitted to Physical Chemistry Chemical Physics. September $22^{\text {th }}, 2014$.

supports well the two-population assumption evoked above. The other two, at $171 \mathrm{~cm}^{-1}$ and 221 $\mathrm{cm}^{-1}$, belong to the connectivity band and are located along each side of the band. It may only reflect a general broadening of the band remaining centered at the same frequency and cannot express the changes expected above $310 \mathrm{~K}$, with a dominant mode of the less-bonded molecules.

\section{DISCUSSION}

\subsection{Modification of the model}

It follows from previous considerations that the model needs to take into account the intramolecular modes, by including the bending and also the stretching through its three sub-bands whose behavior account for the thermal changes in water. The new partition function integrates these modes through the $f_{i}$ factors, which stand for the molecular fractions of each stretching subbands. Thus, in contrast to the original model, these factors are not any more adjustable parameters but become experimental variables, implying that the temperature dependence of the water vibrational modes, expressed by the bands shape changes, can be implemented in the modified model.

Another limitation of the original model is the assumption made by Griffith and Sheraga ${ }^{27}$ that the three-bonded population predominates over the four-bonded population of water molecules at odds with the general consensus that the four-bonded population predominates in water up to $100^{\circ} \mathrm{C}^{3,32}$. Our modified model correctly matches the usual four-bonded dominance.

The new partition function writes as follows:

$$
Z=\sum_{N_{i}} g\left[\prod_{i=1}^{3}\left[f_{i, \text { inter }} \exp \left(-E_{i} / R T\right)\right]^{x_{i} N_{A}} \prod_{i=1}^{3}\left[f_{i, \text { intra }} \exp \left(-E_{i} / R T\right)\right]^{x_{i} N_{A}}\right]
$$

The OH-stretching sub-bands are ascribed to the three types of water molecules (MW, IW, NW) and so the combinatorial factor $\mathrm{g}$ is revised as: $g=\frac{N_{A} !}{N_{N W} ! N_{I W} ! N_{M W} !}$, where $N_{N W}, N_{I W}$ and $N_{M W}$ are respectively equal to $N_{A} x_{N W}, N_{A} x_{I W}$ and $N_{A} x_{M W}$. 
The form of the $f_{i}$ factors was also modified, by using three intermolecular $f_{i}$ factors and three intramolecular $f_{i}$ factors. Each of the three factors is ascribed to one class of water molecules. The different $f_{i}$ factors are given by:

$$
\begin{aligned}
& f_{i, \text { inter }}=\left[1-\exp \left(-h v_{i}^{(T)} / k T\right)\right]^{-3}\left[1-\exp \left(-h v_{i}^{(L)} / k T\right)\right]^{-3} \\
& \left.f_{i, \text { intra }}=\left[1-\exp \left(-h v_{i}^{(B)} / k T\right)\right]^{-3}\left[1-\exp \left(-h v_{i}^{(S)}\right) / k T\right)\right]^{-3}
\end{aligned}
$$

Where $i=1,2,3$ respectively attributed to NW, IW, MW. The frequency $v_{i}^{(B)}$ is ascribed to the bending mode, and $v_{i}^{(S)}$ to the stretching mode. The frequencies of the associated $f_{i}$ factors and their dependency with temperature are presented in Table 2 . The bending mode is hardly sensitive to the environmental conditions, including temperature, and so the band frequency is constant for all classes of molecules in the probed temperature range. The libration band was fitted by two Gaussian components (Gauss 2 and 3) but they have varying width with $T$ preventing their use for extrapolation. Therefore, only the main frequency of the complete band is fitted with $T$ according to a linear dependency. Meanwhile, the connectivity band has only one Gaussian component, linearly varying with $T$ (Table 2).

Table 2. Values of the vibrational characteristic frequencies used in the IR-to-thermodynamic model. Data are either directly measured or calculated with an empirical $v-T$ relationship defined

\begin{tabular}{|c|c|c|c|c|c|c|c|}
\hline $\begin{array}{c}\text { Temperature } \\
(\mathrm{K})\end{array}$ & \multicolumn{3}{|c|}{$v_{i}^{(\mathrm{S})}\left(\mathrm{cm}^{-1}\right)$} & $v_{i}^{(B)}\left(\mathrm{cm}^{-1}\right)$ & $v_{\mathrm{i}}^{(\mathrm{T})}\left(\mathrm{cm}^{-1}\right)$ & \multicolumn{2}{|c|}{$v_{\mathrm{i}}^{(\mathrm{L})}\left(\mathrm{cm}^{-1}\right)$} \\
\hline & NW & IW & MW & \multirow{3}{*}{$\begin{array}{c}1648.84 \\
\mathbf{1 6 5 0}\end{array}$} & Gauss 1 & Gauss & Gauss 3 \\
\hline 263 & 3307.4 & 3464.1 & 3572.5 & & $193.4^{*}$ & \multicolumn{2}{|c|}{$738.7 * *$} \\
\hline 267 & $3305.1^{\dagger}$ & $3457.2^{\dagger \dagger}$ & $3579.3^{\dagger \dagger \dagger}$ & & 192.3 & 574.7 & 755 \\
\hline 268 & 3298.7 & 3451.8 & 3567.9 & 1654.62 & 191.9* & \multicolumn{2}{|c|}{ 735.7 $* *$} \\
\hline 270 & $3305.4^{\dagger}$ & $3^{3457.8} .^{\dagger \dagger}$ & $3580.1^{\dagger \dagger \dagger}$ & $1650^{t}$ & 191.4 & 568.9 & 751.7 \\
\hline 272 & $3305.6^{\dagger}$ & $3458.2^{\dagger \dagger}$ & $3580.6^{\dagger \dagger \dagger}$ & 1650 & 190.4 & 566 & 749.7 \\
\hline 273 & 3302.6 & 3454 & 3573.3 & 1648.84 & $190.5^{*}$ & \multicolumn{2}{|c|}{ 732.7 $* *$} \\
\hline
\end{tabular}
from lower-T measurements (reported in footnotes). 
Revised version of the ID CP-ART-08-2014-003466. Submitted to Physical Chemistry Chemical Physics. September $22^{\text {th }}, 2014$

\begin{tabular}{|c|c|c|c|c|c|c|c|}
\hline 275 & 3303.1 & 3457.6 & 3593.4 & 1651.7 & 190 & 557.3 & 747.8 \\
\hline 277 & $3306.2^{\dagger}$ & $3459.3^{\dagger \dagger}$ & 3581.9 $9^{\dagger \dagger \dagger}$ & $1650^{\ddagger}$ & 189 & 558.3 & 746.3 \\
\hline 278 & 3309.5 & 3464.9 & 3580.1 & 1650.77 & $189.6^{*}$ & \multicolumn{2}{|c|}{ 729.6** } \\
\hline 280 & 3310.1 & 3459.1 & 3592.9 & 1650.8 & 188.5 & 554.4 & 743.9 \\
\hline 283 & 3308.9 & 3466.5 & 3583.6 & 1652.7 & 187.5 & 540.5 & 740.5 \\
\hline 285 & 3307. ${ }^{\dagger}$ & $3461^{\dagger \dagger}$ & 3583.9 $9^{\dagger \dagger \dagger}$ & $1650^{*}$ & 187.1 & 530.3 & 739.1 \\
\hline 288 & 3308 & 3458.4 & 3578.3 & 1650.8 & 186.6 & 527.9 & 738.1 \\
\hline 290 & 3311.4 & 3460.8 & 3594.1 & 1649.8 & $185.6^{*}$ & \multicolumn{2}{|c|}{$722.4 * *$} \\
\hline 291 & $3307.6^{\dagger}$ & $3462.3^{\dagger \dagger}$ & $3585.5^{\dagger \dagger \dagger}$ & 1650 & 185.1 & 508.2 & 730.9 \\
\hline 293 & 3308.1 & 3463.8 & 3583.8 & 1648.8 & 184.7 & 470.1 & 720.8 \\
\hline 296 & $3308.1^{\dagger}$ & $3463.3^{\dagger \dagger}$ & $3586.8^{\dagger \dagger \dagger}$ & $1650^{\ddagger}$ & 183.7 & 460.9 & 710.4 \\
\hline 298 & 3303.9 & 3462 & 3583.7 & 1652.7 & $183.3^{*}$ & \multicolumn{2}{|c|}{ 717.6** } \\
\hline 299 & $3308.4^{\dagger}$ & $3463.9^{\dagger \dagger}$ & $3587.5^{\dagger \dagger \dagger}$ & $1650^{\ddagger}$ & 182.7 & 456.6 & 714.5 \\
\hline 300 & 3312.6 & 3463.3 & 3596.2 & 1648.8 & $182.7^{*}$ & \multicolumn{2}{|c|}{$716.4 * *$} \\
\hline 302 & $3308.7^{\dagger}$ & $3464.5^{\dagger \dagger}$ & $3588.3^{\dagger \dagger \dagger}$ & $1650^{\ddagger}$ & 182.4 & 432 & 703.4 \\
\hline 303 & 3308.9 & 3467.2 & 3587.8 & 1648.8 & 181.8* & \multicolumn{2}{|c|}{ 714.6** } \\
\hline 308 & 3308.4 & 3464.2 & 3586.7 & 1650.8 & $180.4^{*}$ & \multicolumn{2}{|c|}{ 711.6** } \\
\hline 310 & 3312.6 & 3464.5 & 3595.7 & 1647.9 & $179.8^{*}$ & \multicolumn{2}{|c|}{$710.4 * *$} \\
\hline 313 & 3305.3 & 3463.7 & 3586.7 & 1650.8 & 178.9* & \multicolumn{2}{|c|}{ 708.6** } \\
\hline 318 & 3309 & 3472 & 3594.8 & 1652.7 & $177.5^{*}$ & \multicolumn{2}{|c|}{ 705.6** } \\
\hline 320 & 3312.1 & 3467.2 & 3598.1 & 1647.9 & $176.9 *$ & \multicolumn{2}{|c|}{ 704.4** } \\
\hline 323 & 3310.5 & 3468.3 & 3591.9 & 1646.9 & 176.1* & \multicolumn{2}{|c|}{ 702.6** } \\
\hline 328 & 3307.6 & 3467.7 & 3591.7 & 1648.8 & $174.6^{*}$ & \multicolumn{2}{|c|}{$699.5 * *$} \\
\hline 330 & 3315.4 & 3469.3 & 3599.7 & 1646.9 & $174.0^{*}$ & \multicolumn{2}{|c|}{$698.3 * *$} \\
\hline 333 & 3308.9 & 3473.9 & 3597.6 & 1648.8 & $173.2^{*}$ & \multicolumn{2}{|c|}{$696.5 * *$} \\
\hline 338 & 3316.4 & 3476.8 & 3600 & 1646.9 & $171.7^{*}$ & \multicolumn{2}{|c|}{$693.5 * *$} \\
\hline 340 & 3315.3 & 3471.7 & 3601.7 & 1646.9 & 171.1* & \multicolumn{2}{|c|}{$692.3 * *$} \\
\hline 343 & 3309.7 & 3471.6 & 3594.5 & 1650.7 & $170.3^{*}$ & \multicolumn{2}{|c|}{$690.5 * *$} \\
\hline 348 & 3314 & 3478.9 & 3599.5 & 1648.8 & $168.8^{*}$ & \multicolumn{2}{|c|}{$687.5 * *$} \\
\hline 350 & 3317 & 3475 & 3603.7 & 1646 & $168.3^{*}$ & \multicolumn{2}{|c|}{$686.3 * *$} \\
\hline 353 & 3308.9 & 3472 & 3596.8 & 1648.8 & $167.4^{*}$ & \multicolumn{2}{|c|}{$684.5 * *$} \\
\hline 358 & 3314.7 & 3477.6 & 3599.1 & 1648.8 & $165.9 *$ & \multicolumn{2}{|c|}{$681.5 * *$} \\
\hline 363 & 3315.5 & 3476.9 & 3600.2 & 1646.9 & $164.5^{*}$ & \multicolumn{2}{|c|}{$678.5 * *$} \\
\hline
\end{tabular}
assumed constant at $1650 \mathrm{~cm}^{-1} \cdot *: v_{i}^{(T)}=269.4-0.289 T(K) . * *: v_{i}^{(L)}=897.0-0.602 T(K)$.

For data recorded at ambient pressure with laboratory sources, the connectivity band and the low frequency part of the libration band were not recorded. For these measurements, the model is downgraded and only includes the libration main frequency $v_{i}^{(L)}$ (ignoring the two sub-bands behavior), varying linearly with $T$ as extracted from the data (Table 2). For the connectivity band, the same treatment applies, and the calculation then involves a constant main frequency with temperature, with intensity assumed to change linearly with temperature (Table 2). This 
simplification allowed us to extrapolate the IR-to-thermodynamics conversion over the larger range of temperature retained in this study (see below, section 5.2).

To sum up, from $267 \mathrm{~K}$ to $302 \mathrm{~K}$ (experiment 2.3) the model used experimental frequencies for the four bands. For the experiments 2.1 and 2.2 the connectivity band is not available and the libration band is recorded only for its higher frequencies.

Last point, in the model, an energy level must be ascribed to each water species. In the original model, this energy level per species depends on the coordination number of the different water populations. In our modified model, the NW (four-bonded molecules) is at the ground state, i.e. is equal to zero. The energy attributed to the IW state is the energy necessary to transform one O$\mathrm{H}$... O bond in ice into one free $\mathrm{OH}$, estimated to be equal to $E_{b}=23.3 \mathrm{~kJ}_{\mathrm{mol}}{ }^{-1,3,51}$. At last, the energy assigned to the MW should be a combination of the energies needed to break two, three and four H-bonds (so that to produce 2-, 1- and 0-bonded molecules), with the form $x_{2} 2 E_{b}+x_{1} 3 E_{b}+$ $x_{u} 4 E_{b}$. Nevertheless, the molecular fractions of 2-, 1- and 0-bonded molecules are indistinctly covered in the MW fraction. A reasonable assumption is that, in MW, the 2-bonded molecules are the dominating population ${ }^{50,52-54}$, and consequently the energy attributed to the MW state is equals $2 E_{b}$

\subsection{Macroscopic Gibbs free energy from IR data}

The partition function $Z$ is developed from the Maxwell-Boltzmann distribution what makes it a characteristic property of the system at constant temperature and volume. As a consequence, the thermodynamic function directly related to $Z$ is the Helmholtz free energy $F$, the thermodynamic potential at constant $(T, V)$, can be written:

$F=-k T \ln (Z)$

The Gibbs free energy can be obtained from the usual $G=F+P V$ relation, writing:

$G=-k T \ln Z+k T V\left(\frac{\partial \ln Z}{\partial V}\right)_{T}$ 
In the present conditions of the experiment, the correction from Helmholtz to Gibbs free energy (included in our calculations) is practically negligible, amounting to about $2 \mathrm{~J} \cdot \mathrm{mol}^{-1}$ over the whole temperature range $263 \mathrm{~K}-363 \mathrm{~K}$.

From $267 \mathrm{~K}$ to $302 \mathrm{~K}$ (experiment 2.3 ) the vibrational Gibbs free energy well matches the bulk value of the reference $G^{55}$ (Fig 4), calculated as the integral from $0 \mathrm{~K}$ up to $T$ of the ratio $H / T^{2}, H$ the enthalpy itself calculated through the integral of the heat capacity $C p$ along the $0 \mathrm{~K}-T$ range. The data match well with slight deviation as the $T$ goes up $\left(d G \approx 37 \mathrm{~J} \cdot \mathrm{mol}^{-1}\right.$ at $296 \mathrm{~K}$ and $d G=32 \mathrm{~J} \cdot \mathrm{mol}^{-1}$ at $302 \mathrm{~K})$. As introduced above, beyond $302 \mathrm{~K}$, we simplified the model working with the main libration frequency and a linear thermal behavior for the connectivity and libration bands. This simplified conversion still gives a good match, at least up to $318 \mathrm{~K}$.

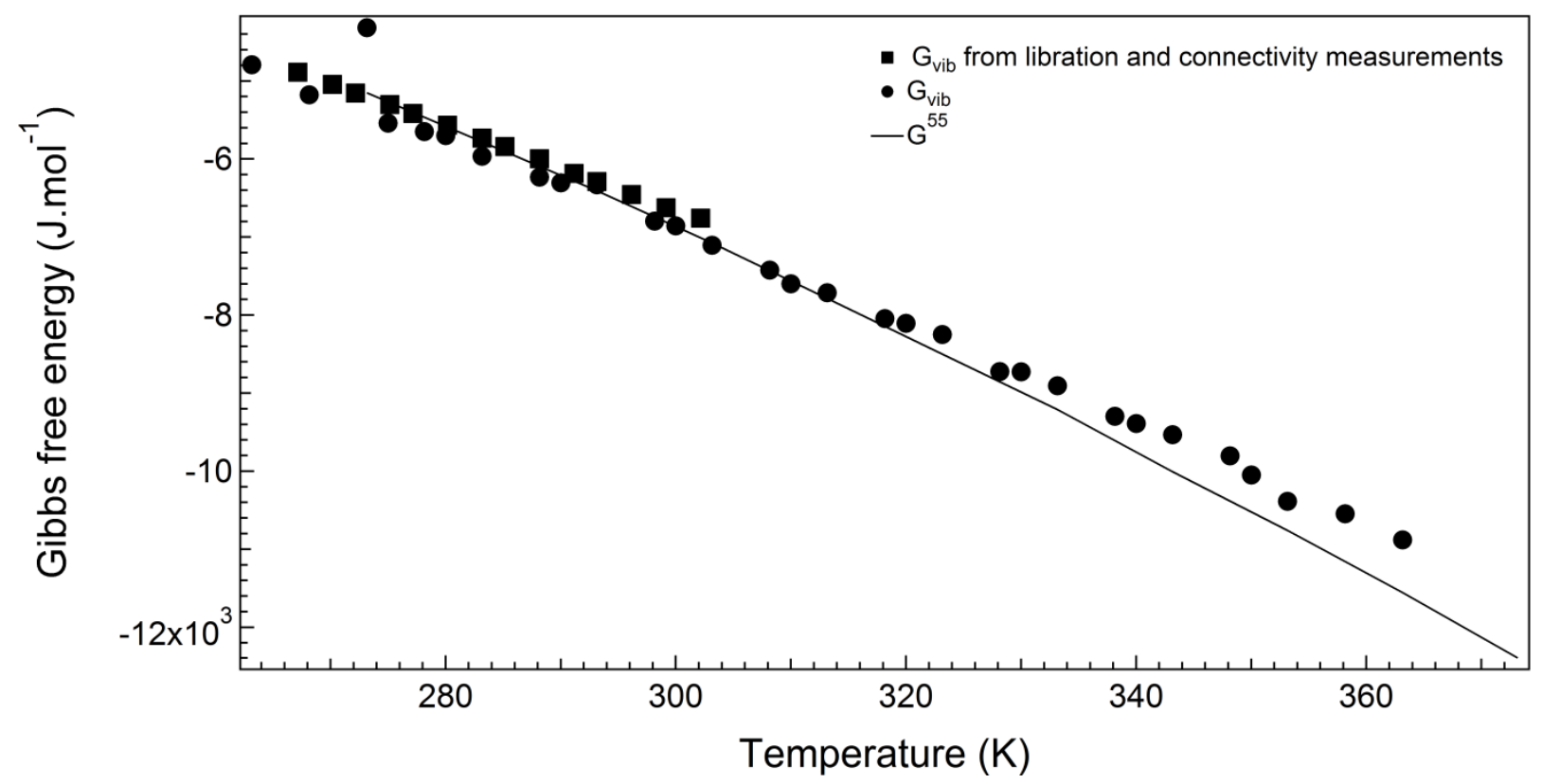

Figure 4. Gibbs free energy of liquid water as a function of temperature deduced from the present measurements and using the partition function described above in the text, compared to reference values $^{55}$. Square: Gibbs free energy calculated from a complete data set of IR bands. Circle: Gibbs free energy calculated from the downgraded model.

Above $318 \mathrm{~K}$ the experimental values of $G$ deviate systematically from the expected values. This deviation may mean that the vibrational part of the partition function becomes insufficient to 
describe correctly the thermodynamics of the system at these higher temperatures. It is interesting to point out that this deviation takes place at the same $T$ at which the isobestic point starts to move significantly with $T$ (see below, section 5.4).

\subsection{Sensitivity of the model}

In this section, some input parameters are modified to test the sensitivity of the output values to the model structure. First, the conversion is performed assuming that the molecular fractions of the three water populations are constant and equal to their values at $298 \mathrm{~K}$. It comes to overlooking the OH-stretching band shape changes. In this case, the conversion is less satisfying (Fig. 5), by 201 $\mathrm{J}_{\mathrm{mol}}{ }^{-1}$ with respect to the value coming from the full calculation. This test supports our hypothesis that decomposing the $\mathrm{OH}$-stretching band gives important information into the energetics of liquid water.

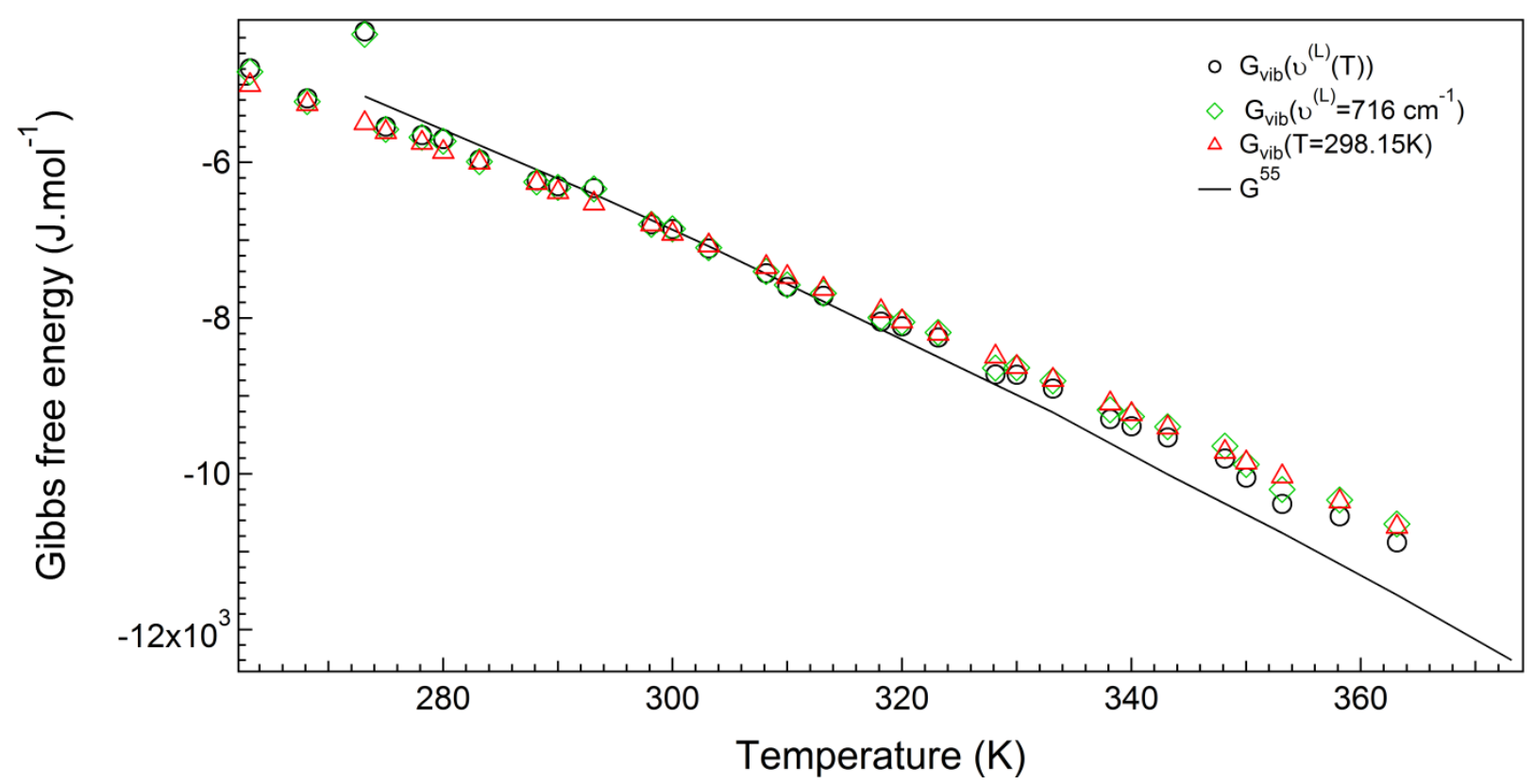

Figure 5. Gibbs free energy as a function of the temperature for molecular fractions fixed at $298 \mathrm{~K}$ (red triangle) and for libration frequency fixed at $716 \mathrm{~cm}^{-1}$ (green lozenge).

Secondly, the influence of the temperature dependence of the frequency of the libration is analyzed comparing the calculations with libration $T$-dependent frequencies with those calculated for a libration fixed frequency (the $298 \mathrm{~K}$ one). The former gives a better agreement with the reference 
Revised version of the ID CP-ART-08-2014-003466. Submitted to Physical Chemistry Chemical Physics. September $22^{\text {th }}, 2014$

values $\left(-10643 \mathrm{~J} \cdot \mathrm{mol}^{-1}\right.$ at $363 \mathrm{~K}$, instead of $-10879 \mathrm{~J} \cdot \mathrm{mol}^{-1}$ ), even if the correction is quite small (less than $50 \mathrm{~J} . \mathrm{mol}^{-1}$ ) below $318 \mathrm{~K}$.

These sensitivity tests demonstrate that the IR spectra of water provide enough information to precisely calculate its thermodynamic properties. To reach a fine tuning, and so to follow minor variations of thermodynamics with temperature, precise measurements and an upgraded description of each band must be done. This was realized using the synchrotron radiation data measured in the 263K-302K range.

Finally, the model assumes that all the water molecules are in the fundamental energy state when they are probed by the infrared beam. From the point of view of an isolated molecule, the Boltzmann distribution predicts that only a small proportion will be in an excited bending state (close to $0.1 \%$ ) or even less so in an excited stretching state. However, if one consider the water network as a super molecule with their collective modes (connectivity and libration) there is a strong probability for the super molecules to be in an initial excited state following Boltzmann distribution. At room temperature, close to $40 \%$ of the super molecules have their H-bond stretching excited in $\mathrm{v}=1$ at room temperature and $2 \%$ of the frustrated rotation in its first excited state. For the present calculation, we used the Boltzmann law to at $300 \mathrm{~K}$ and a connectivity main frequencies at $200 \mathrm{~cm}^{-1}$ to determine the proportion of molecules in an excited energy level at about 0.3805 . The effect of the excited initial state on the model modifies the connectivity part of the $f_{i, \text { inter }}$ to take into account the proportion of the excited molecules. At $302 \mathrm{~K}$, the Gibbs free energy using this modification equals $-6747.45 \mathrm{~J} . \mathrm{mol}^{-1}$, that is to say a negligible variation of $0.22 \%$.

\subsection{Isobestic point and thermodynamics}

As argued before ${ }^{44}$, the isobestic points constitute a strong evidence for a mixture model of water involving the general $\mathrm{H}$-bonded and non $\mathrm{H}$-bonded classes of $\mathrm{OH}$ oscillators. It is interesting to note that Griffiths and Scheraga developed their partition function on such grounds. Alternatively, the isobestic point has been defined ${ }^{31}$ as a frequency barycenter between molecules that participate to 
the extended connective networks and those lying outside these networks. Almost all molecules are assumed to be engaged into H-bonds, but here the level of network connectivity makes the difference between two populations. The present measurements only provide the mean field properties connected to the statistical connectivity in the water network, therefore supporting the latter interpretation of the isobestic point in terms of network connectivity. This analysis is also supported by the presence of isobestic points at low frequencies, in the intermolecular domain.

The laboratory measurements in the MIR above $310 \mathrm{~K}$, show a loss of the isobestic point in the $\mathrm{OH}$ stretching band. This deviation suggests a growing impact of the translational and rotational components in the water energetics, owing to the increasing number of isolated molecules (increasing intensity of the MW band). As a consequence, the frequencies barycenter of the water network is no more balanced between two vibrational populations, but one population combines more modes than the other: the isobestic point has no physical meaning anymore. In this description, the disappearance of the isobestic point simply indicates that the vibrational energy no more features the whole thermodynamic properties of the liquid.

\subsection{Vibrational and total Gibbs free energy}

Generally speaking, one partition function is written as the product of different contributions: translational, electronic, nuclear, rotational and vibrational components. For liquid water, the nuclear and electronic partitions functions are equal to $1^{56}$, while the translational and rotational contributions are given by:

$$
\begin{aligned}
& f_{\text {trans }}=\left(\frac{2 \pi m k T}{h^{2}}\right)^{3 / 2} V \\
& f_{\text {rot }}=\frac{2 \pi}{\sigma}\left(\frac{2 \pi I k T}{h^{2}}\right)^{1 / 2}
\end{aligned}
$$

With $m$ the mass of the molecule, $V$ is the volume available to the translation motions, $\sigma=2$ the symmetry number of water and $I$ the inertia moment of the molecule. For highly connected molecules, the rotational and translational motions are negligible, as expected in liquid water at low 
to moderate temperatures. When temperature goes up, translational and rotational effects may come into play so that $f_{\text {trans }}$ and $f_{\text {rot }}$ should be weighted by the molecular fraction of 0-bonded molecules. Therefore, one can estimate the $x_{0}$ molecular fraction by matching the calculated and the theoretical $G$, through a calculation that includes weighted $f_{\text {trans }}$ and $f_{\text {rot }}$. The percentage of 0 bonded molecules thus calculated is $4.5 \%$ at $363 \mathrm{~K}$, indeed highly consistent with other studies ${ }^{37-39}$. The vibrational Gibbs free energy including rotational and translational terms can match the Dorsey reference values ${ }^{55}$ through a reasonable estimate of the unbounded molecules. Vibrational energy may not be able to fully describe all the energy state reached by liquid water in the full range of liquid water temperature, but seem adapted to describe the thermodynamics properties of water in a large domain of temperature. Table 3 shows the ratio between the vibrational energy and the total Gibbs free energy. From 263 to $310 \mathrm{~K}$, the ratio $G_{v i b} / G_{t o t}$ approaches 1 and then decreases slightly, as already discussed above. In our present interpretation, this deviation trend is a clue that the rotational and translational components come into play.

Table 3, Vibrational, total Gibbs free energy and ratio of $G_{v i b} / G_{t o t}$ as a function of the temperature. The grey cells report Gibbs free energies calculated from our complete dataset over all IR bands and the white cells report Gibbs free energies calculated from the downgraded model.

\begin{tabular}{|c|c|c|c|}
\hline Temperature (K) & $G_{v i b}\left(\mathrm{~J} \cdot \mathrm{mol}^{-1}\right)$ & $G_{\text {tot }}\left(\mathrm{J}_{\mathrm{mol}} \mathbf{\mathrm { l }}^{-1}\right)$ & $G_{v i b} / G_{t o t}\left(\mathrm{~J} \cdot \mathrm{mol}^{-1}\right)$ \\
\hline 263 & -4791.88 & -4608.46 & 1.04 \\
\hline 268 & -5175.37 & -5175.37 & 1.00 \\
\hline 270 & -5030.05 & -4857.05 & 1.04 \\
\hline 275 & -5289.41 & -5211.1 & 1.01 \\
\hline 277 & -5396.05 & -5352.71 & 1,01 \\
\hline 280 & -5546.12 & -5565.14 & 1 \\
\hline 283 & -5721.35 & -5777.57 & 0.99 \\
\hline 285 & -5836.39 & -5919.19 & 0.99 \\
\hline 288 & -5988.9 & -6131.61 & 0.99 \\
\hline 290 & -6304.30 & -6193.28 & 1.02 \\
\hline 293 & -6400.17 & -6485.66 & 0.98 \\
\hline 296 & -66601.97 & -6698.09 & 0.99 \\
\hline 298 & -6794.66 & -6784.42 & 1.00 \\
\hline 300 & -6856.82 & -6918.60 & 0.99 \\
\hline 302 & -7042.75 & -7122.91 & 0.99 \\
\hline
\end{tabular}


Revised version of the ID CP-ART-08-2014-003466. Submitted to Physical Chemistry Chemical Physics. September $22^{\text {th }}, 2014$.

\begin{tabular}{l|l|l|l}
308 & -7421.49 & -7509.74 & 0.99 \\
310 & -7598.14 & -7643.92 & 0.99 \\
318 & -8042.66 & -8235.06 & 0.98 \\
320 & -8105.86 & -8369.24 & 0.97 \\
328 & -8721.66 & -8960.38 & 0.97 \\
330 & -8724.33 & -9094.56 & 0.96 \\
338 & -9295.07 & -9685.70 & 0.96 \\
340 & -9388.53 & -9819.88 & 0.96 \\
350 & -10047.60 & -10545.20 & 0.95 \\
353 & -10385.10 & -10773.70 & 0.96
\end{tabular}

\section{CONCLUSIONS}

IR measurements have been performed on liquid water, from supercooling state up to $363 \mathrm{~K}$, recording the evolution of the connectivity, libration, bending and $\mathrm{OH}$ stretching bands as a function of temperature. The recorded trends are consistent with previous studies, either experiments or simulations. Among all bands, the stretching band appears as the most sensitive. This justifies the intense study of this spectral range, which offers interesting prospect to investigate other types of water such as confined, capillary, interfacial, or solvated water ${ }^{18,23,40}$. The $\mathrm{OH}$ stretching bands measured through two different setups are consistent with each other and in agreement with previous studies. A decreasing connectivity on the mean water network builds up as the temperature increases and comes closer to the boiling point. However, the decomposition of the band into three Gaussian components demonstrates that the network remains energetically favorable to molecules that are tetrahedrically bonded.

The IR-to-thermodynamics conversion is well validated by its ability to retrieve the well-known Gibbs free energy, in this thermal range. The modified partition function is able to describe the

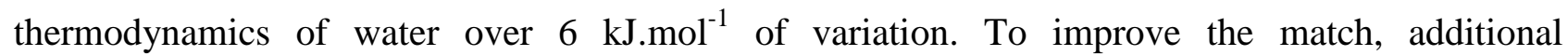
contributions are required above $318 \mathrm{~K}$. The disappearance of the isobestic point with temperature, concomitantly with the thermodynamic deviation, is understood as another indication that the IR vibrational bands feature only part of the thermodynamics of water. Nevertheless, the vibrational 
Revised version of the ID CP-ART-08-2014-003466. Submitted to Physical Chemistry Chemical Physics. September $22^{\text {th }}, 2014$.

Gibbs free energy reproduces satisfactorily the thermal induced change in a large domain of temperatures for the liquid state.

Promising future studies involve recording the infrared features of liquid water trapped in different containers to probe if and how the thermodynamic properties are modified in porous networks.

Acknowledgements. This work has received financial support from the French Agency for Research (Agence Nationale de la Recherche), grant CONGE ANR-2010-BLAN-0610 and from Region Centre grant 201100070577 SIRE. It also benefits from the SOLEIL Synchrotron allocation $\mathrm{n}^{\circ}$ 99130036. At last, the support of labex Voltaire (grant ANR-10-LABX-100-01) is also acknowledged.

\section{REFERENCES}

[1] D. Eisenberg and W. Kauzmann, in The structure and properties of water, clarendon Press, Oxford, 1969, 296p.

[2] B. Cabane and R. Vuilleumier, C. R. Geoscience, 2005, 337, 159-171.

[3] Y. Maréchal, in The Hydrogen Bond and the Water Molecule: The Physics and Chemistry of Water, Aqueous and Bio Media, Elsevier, Amsterdam, 2007, 318p.

[4] Y. Marcus, Pure Appl. Chem., 2010, 82(10), 1889-1899.

[5] H. Ohtaki and T. Radnai, Chem. Rev., 1993, 93, 1157-1204.

[6] B.V. Derjaguin, N.V. Churaev and V.M. Muller,in Surface forces. Plenum Publishing Corporation, New York, 1987.

[7] L.J. Michot, F. Villiéras, M. François, I. Bihannic, M. Pelletier and J.-M. Cases, C.R. Geoscience, 2002, 334, 611-631.

[8] R. Akiyama and F. Hirata, J. Chem. Phys., 1998, 108, 4904-4911.

[9] J. Dore, Chem. Phys., 2000, 258, 327-347. 
Revised version of the ID CP-ART-08-2014-003466. Submitted to Physical Chemistry Chemical Physics. September $22^{\text {th }}, 2014$

[10] J. Teixeira, J.-M. Zanotti, M.-C.Bellissent-Funel and S.-H. Chen, Physica B, 1997, 234-236, 370-374.

[11] Y.P. Handa, M. Zakrzewski and C. Fairbridge, J. Phys. Chem., 1992, 96, 8594-8599.

[12] P. Fenter and N.C. Sturchio, Prog. Surf. Sci., 2004, 77, 171-258.

[13] F.O. Libnau, O.M. Kvalheim, A. A. Christy and J. Toft, Vib. Spectrosc., 1994, 7, 243-254.

[14] Y. Maréchal, J. Chem. Phys, 1991, 95, 5565.

[15] Y. Maréchal, J. Mol. Struct., 2011, 1004, 146-155.

[16] L. Mercury, F. Jamme and P. Dumas, Phys. Chem. Chem. Phys., 2011, 14, 2864-2874.

[17] T. Richard, L. Mercury, F. Poulet and L. d'Hendecourt, J. Colloid Interf. Sci., 2006, 304, 125136.

[18] C. Boissière, J.-B. Brubach, A. Mermet, G. de Marzi, C. Bourgaux, E. Prouzet, and P. Roy, J. Phys. Chem. B, 2002, 106, 132-135.

[19] H. MacDonald, B. Bedwell and E. Gulari, Langmuir, 1986,2(6), 704-708.

[20] G. Onori and A. Santucci, J. Phys. Chem, 1993, 97, 5430-5434.

[21] H.A. Abadleh and V.H. Grassian, Langmuir, 2003,19, 341-347.

[22] M.R. Yallamanchili, A.A. Atia and J.D. Miller, Langmuir, 1996, 12, 4176-4184.

[23] K. Masuda, H. Taishiro, S. Nakashima, B. Habert, I. Martinez and S. Kashiwabara, Appl. Spectrosc., 2003, 57(3), 274-281.

[24] Y.Y. Efimov and Y.I. Naberukhin, Mol. Phys., 2003, 101(3), 459-468.

[25] Y.Y. Efimov and Y.I. Naberukhin, Mol. Phys., 2004, 102(13), 1407-1414.

[26] Y.Y. Efimov and Y.I. Naberukhin, Spectrochim. Acta A, 2004, 61, 1789-1794.

[27] J.H. Griffith and H.A. Scheraga, J. Mol. Struct. (Theochem), 2004, 682, 93-113.

[28] G. Némethy and H.A. Scheraga, J. Chem. Phys., 1962, 36, 3382-3400.

[29] H.S. Frank and W.-Y. Wen, Discuss. Faraday.Soc., 1957, 24, 133.

[30] H.E. Stanley and J. Teixeira, J. Chem. Phys., 1980, 73(7), 3404-3422. 
Revised version of the ID CP-ART-08-2014-003466. Submitted to Physical Chemistry Chemical Physics. September $22^{\text {th }}, 2014$

[31] J.-B. Brubach, A. Mermet, A. Filabozzi, A. Gerschel and P. Roy, J. Chem. Phys., 2005, 122, 184509.

[32] F.H. Stillinger, Science, 1980, 209(4455), 451-457.

[33] V. Mazet, C. Carteret, D. Brie, J. Idier and B. Humbert, Chemom. Intell. Lab. Syst., 2005, 76(2), 121-133.

[34] M. Praprotnik, D. Janezic, and J. Mavri, J. Phys. Chem. A, 2004, 108, 11056-11062.

[35] H.R. Zelsmann, J. Mol. Struct., 1995, 350, 95-114.

[36] K. Nakamoto, M. Margoshes and R.E. Rundle, J. Am. Chem. Soc., 1955, 77(24),6480-6486.

[37] M. Freda, A. Piloso, A. Santucci, and P. Sassi, Appl. Spectrosc., 2005, 59(9), 1155-1159.

[38] B. Czarnik-Matusewicz, S. Pilorz and J.P. Hawranek, Anal. Chim. Acta., 2005, 544, 15-25.

[39] N.V. Nucci and J.M. Vanderkooi, J. Phys. Chem. B., 2005, 109, 18301-18309.

[40] S. Le Caër, S. Pin, S. Esnouf, Q. Raffy, J.Ph. Renault, J.-B. Brubach, G. Creff and P. Roy, Phys. Chem. Chem. Phys., 2011, 13, 17658-17666.

[41] P. Jedlovsky, I. Bako and G. Palinkas, Chem. Phys. Lett., 1994, 221, 183-187.

[42] R. Kumar, J. R. Schmidt and J.L. Skinner, J. Chem. Phys., 2007, 126, 204107.

[43] G.V. Lagodzinskaya, N.G. Yunda and G.B. Manelis, Chem. Phys., 2002, 282, 51-61.

[44] G.E. Walrafen, M.S. Hokmabadi and W.-H.Yang, J. Chem. Phys., 1986, 85, 6964-6969.

[45] G. D’Arrigo, G. Maisano, F. Mallamace, P. Migliardo, and F. Wanderlingh, J. Chem. Phys., 1981, 75, 4264-6270.

[46] G.E. Walrafen, M. R. Fisher, M. S. Hokmabadi and W.H. Yang, J. Chem. Phys.,1986, 85, 6970-6982.

[47] P.L. Geissler, J. Am. Chem. Soc., 2005, 127(42), 14930-14935.

[48] J.-J. Max and C. Chapados, J. Chem. Phys., 2002, 116, 4626.

[49] P. Larouche, J.-J.Max and C. Chapados, J. Chem. Phys., 2008, 129, 064503.

[50] S. Thaomola, A. Tongraar and T. Kerdcharoen, J. Mol. Liq., 2012, 174, 26-33. 
Revised version of the ID CP-ART-08-2014-003466. Submitted to Physical Chemistry Chemical Physics. September $22^{\text {th }}, 2014$.

[51] E. Whalley, The hydrogen bond: Recent theory and Experiments, in Schuster, G Zundel and C. Sandorfy (Eds), Vol. 3, Ch. 29, North Holland, Amsterdam, 1976.

[52] F.H. Stillinger and A. Rahman, J. Chem. Phys., 1974, 60, 1545.

[53] A. Luzar, Chem. Phys., 2000, 258, 267-276.

[54] D. Swiatla-Wojcik, Chem. Phys., 2007, 342, 260-266.

[55] Dorsey, N. E., Properties of ordinary water-substance, in all its phases: water-vapor, water and all the ices. American chemical society Monograph Series. New York; Hafner, 1968,673 p.

[56] J.M.L. Martin, J.P. Francois and R. Gijbels, J. Chem. Phys, 1992, 96, 7633. 